\section{Histoire Épistémologie Langage}

42-1 | 2020

La grammaire arabe étendue

\title{
Transitivité et intransitivité dans la grammaire de Bar Hebræus
}

\section{Georges Bohas}

\section{OpenEdition}

1 Journals

\section{Édition électronique}

URL : https://journals.openedition.org/hel/500

DOI : $10.4000 /$ hel. 500

ISSN : 1638-1580

\section{Éditeur}

Société d'histoire et d'épistémologie des sciences du langage

\section{Édition imprimée}

Date de publication : 28 septembre 2020

Pagination : 49-57

ISSN : 0750-8069

\section{Référence électronique}

Georges Bohas, "Transitivité et intransitivité dans la grammaire de Bar Hebræus », Histoire Épistémologie Langage [En ligne], 42-1 | 2020, mis en ligne le 28 octobre 2021, consulté le 29 octobre 2021. URL : http://journals.openedition.org/hel/500 ; DOI : https://doi.org/10.4000/hel.500

Ce document a été généré automatiquement le 29 octobre 2021.

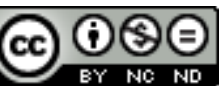

$\mathrm{HEL}$ is licensed under a Creative Commons Attribution-NonCommercial-NoDerivatives 4.0 International License 


\title{
Transitivité et intransitivité dans la grammaire de Bar Hebræus
}

\author{
Georges Bohas
}

\section{Introduction}

1 La propriété «transitif/intransitif » ne figurant pas dans la grammaire de Denys le Thrace ${ }^{1}$, qui, ayant été traduite par Joseph d'Ahwaz (mort en 580), a été le berceau des grammaires syriaques, la tradition syriaque l'a négligée, jusqu'à Bar Hebræus, qui, selon Merx, l'a empruntée à ZamaHš̌arī :

Etiam aliam rem ex grammatica arabica haustam Syriacae adhibuit, distinctionem verbi transitivi, dupliciter transitivi et intransitivi, quam a Zamakhchario depromsit...

Il y a encore une autre chose puisée dans la grammaire arabe qu'il introduisit en syriaque: la distinction verbe transitif, verbe doublement transitif et verbe intransitif, qu'il emprunta à ZamaHšarīi (Merx 1889: 253).

2 L'objet de cette communication est de voir comment la notion a transité de l'arabe au syriaque et comment Bar Hebræus (désormais BH) l'a «acclimatée » dans son modèle. Quant à savoir comment le concept de transitivité s'est élaboré dans la tradition grammaticale arabe jusqu'à ZamaHšarī, cela n'entre évidemment pas dans le cadre de la présente étude.

3 Il n'est pas inutile de mentionner que ZamaHšarī est mort en 1144 et BH en 1286 : plus d'un siècle les sépare donc, mais BH est presque contemporain d'Ibn Ya'ǐš (1158-1245), l'auteur du grand commentaire sur le Mufașsal', puisqu'il est né en 1226. Que BH ait emprunté à ZamaHšarī, illustre l'influence très importante qu'il a connue en Orient arabe et dans le monde iranophone.

Rappelons d'abord que le texte de ZamaHšarī sur la transitivité se trouve p. 257-58 celui de BH se trouve dans le livre 2 consacré au verbe, p.92-95. Dans la notation des exemples syriaques nous négligerons la spirantisation des bgdkpt. Nous adopterons la vocalisation occidentale (jacobite). Comme nous l'avons montré dans Bohas (2008), BH 
traite des lettres et non pas des sons. Nous veillerons donc dans notre transcription à garder les lettres, particulièrement le 'ōlaf que nous transcrirons par'. De plus, nous ne transcrirons pas $\bar{i}$, mais $\bar{i} y$, nous ne transcrirons pas $\bar{e}$, mais $\bar{e} y$ pour garder la voyelle et la lettre et rester cohérent avec sa pensée. Le seul problème concerne le $w$ que nous gardons tel quel lui aussi.

\title{
1 Verbes transitifs et intransitifs
}

\author{
Chapitre I, à propos de l'intransitivité et de la transitivité des verbes : quatre \\ sections.
}

Première section : À propos d'exemples de verbes intransitifs et transitifs

Tout verbe, ou bien ne transite pas du sujet vers l'objet, mais se restreint au sujet ${ }^{3}$ et est appelé intransitif comme 'etō' fetrōws ['Pierre est venu'], 'ezal fōwlōws ['Paul est parti'], npal ywdōo' ['Judas est tombé'], qōm matīya' ['Mattiyas s'est levé'].

Ou bien il transite de l'un à l'autre et est appelé transitif, comme 'aqiym ['il a fait lever'], 'ahet ['il a fait descendre'], 'apeq ['il a fait sortir'], 'a'el ['il a fait entrer'].

Certains ${ }^{4}$ appellent simple un intransitif comme bōt ['il a passé la nuit'] et composé un transitif comme 'abiyt ['il a fait passer la nuit'].

\section{Élucidation}

Tout verbe transitif passe à partir du sujet ou bien vers un objet comme $m h \bar{o} \bar{o}$ mōryo Imeșrōye ${ }_{1}$ ['Le Seigneur a frappé les Égyptiens '] ${ }_{1}$, ou bien vers deux objets comme :

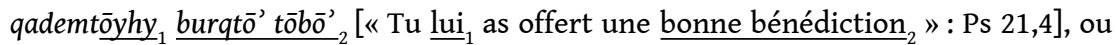

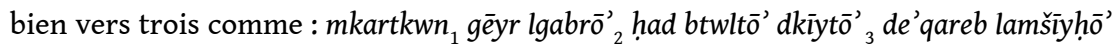
[ "Je vous ai fiancés à un homme (comme) une vierge pure, lequel je présenterai au Christ »: I Cor, 11, 2].

Dans le premier cas, il y a un complément : le nom «Égyptiens »; dans le deuxième cas, le premier complément est le pronom y et ses compagnons [i.e. la séquence oyhy] et le second est: "une bonne bénédiction»; dans le cas de trois compléments, le premier est le pronom $k$ et ses compagnons [i.e. la séquence $k w n$ ], le deuxième est «un homme» et le troisième "une vierge pure». "Que je présenterai au Christ » n'est pas un quatrième complément mais une explication $\mathrm{du}$ deuxième complément à savoir: "un homme». Ainsi, dans la parole: sagiy'e' haymen beh ${ }_{1}$ [ Beaucoup crurent en lui »: Jean, 2, 23], il y a un complément et dans : hw dēyn yešw' lö' mhaymen-hwa lhwn ${ }_{1}$ nawšeh ${ }_{2}$ [" Jésus ne leur $\underline{1}_{1}$ confiait pas son $\underline{\text { âme }}_{2}$ ": Jean, 2, 24], il y a deux compléments [" leur » et « son âme », c'est-à-dire, lui-même, donc pour rendre cela en français : « ne se fiait pas à eux »].

Une première différence avec les grammaires arabes est l'abondance des exemples, particulièrement scripturaires, dont fourmille la grammaire de BH. Comme il n'y a pas de cas apparents en syriaque, contrairement au texte de ZamaHšarī où tous les compléments sont à l'accusatif, et donc les verbes transitent directement, BH ne distingue pas transitif direct $v s$ indirect. Cela est d'autant plus motivé par le fait qu'en syriaque, ce que nous appelons le complément direct est le plus souvent introduit par la préposition 1 (parallèle au 'et de l'hébreu biblique : bāră' 'elohim 'et haššamayim we'et ha'areș [Genèse I, 1]). 


\section{Les causes de la transitivité} transitivité permet de rendre compte de la relation entre la forme simple du verbe et les formes dérivées 'af'ala (la FIV des grammaires arabisantes) et fa "ala (FII). Ces formes ont (généralement pour la première, fréquemment pour la seconde) une valeur causative ou factitive; toutefois, celle-ci est exprimée en termes de transitivité : la préfixation d'un' dans le cas de 'af' ala et le redoublement de la consonne médiane dans celui de fa" ala ont pour effet de rendre transitifs ${ }^{5}$ les verbes intransitifs. ZamaHš̌arī parle à ce propos de « causes de la transitivité » (asbāb al-ta'diya). BH applique la même idée aux formes correspondantes du syriaque: la forme avec préfixe ', $m, n$ ou $t$, équivalente de la forme IV de l'arabe ${ }^{6}$ et la forme avec première consonne mue par un $a$, équivalente de la forme II de l'arabe. Pour la tradition syriaque, c'est en effet cette vocalisation de la première consonne par un a qui distingue FI bșar de FII bașar. On a donc les équivalences suivantes :

FI première consonne quiescente : bșar.

FII première consonne mue par un $\mathrm{a}:$ bașar = forme à redoublement de l'arabe.

FIV préfixation ' $m n t$ : 'aškeb = forme à préfixation de 'a de l'arabe.

Deuxième section : des causes de la transitivité

Par deux causes un verbe intransitif devient transitif.

[1] La première est l'introduction des lettres ' $m n t$, comme

- de škeb ['être étendu'] à 'aškeb ['faire se coucher'] : 'aškeb 'enwn 'al 'ar'ō' ['Il les fit se coucher à terre' : II Sam, 8, 2] ;

- de sțō' ['se détourner'] à 'asțīy ['détourner']: wa'sțīy nešawhy lebeh [«Ses femmes détournèrent son cœur » : I Rois, 11, 3] ;

- de sged ['se courber'] à 'asged ['adorer'] : wfwmeh 'asgdeh galyō'ìyt ['Sa bouche le fit adorer distinctement'] ;

- de hmas ['comprendre'] à 'ahmes ['faire comprendre']: wa'hmes beh kul re'yōniyn ['Il lui fit comprendre tous les sens'] ;

- de $q^{\prime} \bar{o}^{\prime}$ ['crier'] à 'aq '`y ['faire crier']: wke'bō' dhašeh maq'e' lìy ['Et la douleur qu'il ressentit me fit crier'];

- de mak ['être courbé'] à 'amek ['incliner']: wa'mek rišō' lšalìyțō ['Il inclina la tête vers le puissant'] ;

- de hgō' 'réfléchir' à 'ahgīy 'enseigner' : wmanw ktab lōk 'ōlaf bēyt dhō' 'ahge' lōk hafko'ìyt ['Qui t'a écrit l'alphabet ? Car regarde, il t'a enseigné de travers'].

Il en va de même pour $d k a r h w$ ['il s'est souvenu'] et 'adker lahrōnōo' ['il a rappelé à un autre'] : wa'nt dkart 'lay 'awlo' da'nttō' [Mot à mot : 'Tu te souviens contre moi de la faute (ou d'une faute) d'une femme (ou de la femme, ou concernant la femme)' : II Sam, 3, 8] ; tehwe' 'ar' 'o' dīyhwd lmeșrōyō' lswrōdō' wkul dnadkr ìyh leh netrheb ['La terre de Juda deviendra la honte de l'Égypte, chaque fois qu'on la lui rappellera elle sera terrorisée' : Isaïe 19, 17]. 
[2] La deuxième consiste à assigner un $a$ à la première lettre quiescente.

Ainsi, à partir de bșar ['être petit'], gmar ['être complet'], twö' ['s'irriter'], dwīy ['être faible'], hsar ['manquer'] où la première est quiescente, on obtient bașar ['diminuer'], gamar ['achever'], tawì ['gourmander'], dawiy ['affaiblir/frapper ${ }^{9}$ '], hasar ['manquer'] en vocalisant la première avec un $a$

- tawìy: wtawìy dawìy lgabre' d'ameh ['Et David gourmanda les hommes qui étaient avec lui' : I Sam 24, 8] ;

- dawī : dawìt 'enwn bhemty ['Je les ai frappés dans ma fureur'] [Isaïe 63, 6] ;

- hasar : mōry $\bar{o}$ ner'ēyny wmedem lō' nhasar lìy ['Le Seigneur me fait paître et il ne me laisse manquer de rien' : Psaume 23,1].

10 Une remarque s'impose ici. Dans une forme comme bașar, la consonne médiane est en fait géminée, et elle est bien prononcée géminée de nos jours dans la version orientale du syriaque, en revanche dans la version occidentale, il n'y a pas de gémination. $\mathrm{BH}$, comme nous l'avons rappelé ci-dessus, ne prend pas en compte les sons mais bien les lettres, donc pour lui [bașșar], qui est écrit bașar est bien un trilitère, avec vocalisation de la première consonne par un $a$, ce qui l'oppose à la première forme bșar où cette consonne est quiescente. Nous le suivons en cela, pour la cohérence de l'argumentation.

\section{Élucidation}

Parfois l'action transite par le biais d'un mot ou d'une lettre qui indique le complément, comme dans :

- 'etpaniy lwōty wašma' bō'wty ['Il s'est tourné vers (lwōt) moi (y) et il a écouté ma demande'] ;

- we'nō' ldōdy wa 'lay fnoyteh ['Je suis à mon bien-aimé et sur ('al) (=vers) moi (y) son retour (i.e. : il est revenu vers moi)]' : Cantique, 7,11 ];

- wașbayt mōryō’ ba'r'ōk ['Tu t'es complu, Seigneur, dans (b) ta terre' : Ps 85, 2].

Dans ces exemples en effet, c'est par vers lwōt, sur 'al, et dans $b$ qu'ont transité du sujet vers l'objet le fait de se tourner, de revenir et de se complaire.

11 On retrouve ici la troisième «cause de transitivité » mentionnée par ZamaHš̌arī (Mufașșal : 257) : la «transitivité par préposition » (al-ta'diya bi-harf ğarr), par laquelle un verbe intransitif devient transitif. Ainsi Harağtu ('je suis sorti’) devient transitif par l'ajout de la proposition bi- (et acquiert par la même occasion une valeur causative) dans Harağtu bi-hi ('je l'ai fait sortir'), équivalant à 'aHrağtu-hu. Selon ZamaȞšarī, le même procédé peut concerner les verbes transitifs, qui deviennent alors doublement transitifs, ainsi gașabta l-ḍay'ata ('tu t'es emparé de force de la propriété') et ġaṣabta 'alay-hi l-day'ata ('tu l'as spolié de la propriété') :

\section{Élucidation}

Quand une cause de transitivité s'applique à un verbe qui transite [déjà] vers un complément, elle le rend doublement transitif, comme de gbo ' ['choisir'] à 'agbiy ['faire choisir'] : 'agbyan yōrtwteh ['Il nous $\underline{\underline{u}}_{1}$ a laissés choisir son héritage' ${ }_{2}$ : Psaume 47, 5]. De rhem ['aimer'] à 'arhem ['faire aimer'] : yahb lhwn wa'rhem 'enwn l'amme' 
['Il leur a donné et $\underline{\text { les }}_{1}$ a fait aimer $\underline{\text { des peuples }}_{2}$ : Deutéronome, 33, 3]. De šbo '’ ['emmener en captivité'] à 'ašbe' ['faire conduire en captivité', inaccompli] : wa'šbe' šbìteky baynōthêyn ['Je ferai conduire en captivité tes prisonniers ${ }_{1}$ au milieu d'elles' ${ }_{2}$ : Ézéchiel 16, 53]. De s'en ['chausser'] à 'as'en ['faire revêtir des

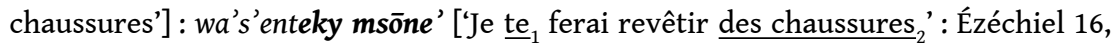
10]. De baz ['piller'] à 'abez ['donner à piller', d'où 'mépriser']: we'n 'ōmrīytwn bmōnō' 'abezn šmōk ['Et si vous dites: en quoi ${ }_{1}$ avons-nous donné à piller (i.e. méprisé) ton nom ${ }_{2}^{\prime}$ : Malachie 1,6]. De 'ehad ['prendre'] à 'ahed ['louer, donner en location'] : wa'whdeh lfalōhe' ['Et il la $\underline{a}_{1}$ loua à des travailleurs ${ }_{2}$ ': Matth. 21, 33 et Luc, $20,9]^{10}$.

\section{L'échec des causes de la transitivité}

BH aborde ensuite les cas où la préfixation de 'mnt (correspondant à la FIV, 'af'ala de l'arabe) et la vocalisation de l'initiale par a (correspondant à la FII, fa 'ala) n'ont rien à voir avec la transitivité, mais sont corrélées à un changement de sens, le second étant tout à fait étranger au premier.

Troisième section : À propos de l'échec des causes de transitivité

L'adjonction des préfixes 'mnt et la vocalisation par $a$ de la première quiescente ne provoquent pas toujours la transitivité. Ainsi les verbes 'azhar ['briller'], 'aḥwar ['être blanc'], 'agreb ['être lépreux'] et 'agah ['briller'] ont bien un préfixe ', $m, n, t$, mais ne sont pas transitifs :

- 'azhar ['briller'] : wmazhar hwō' lbwšeh ['Et ses vêtements brillaient' : Marc, 9, 3] ;

- 'ahwwar ['être blanc'] : wmaḥwar 'ayk talgō' ['et étaient blancs comme neige' : Marc $9,3]$;

- 'agreb ['être lépreux'] w'ìydeh magrbō' 'ayk talgō' ['Sa main était lépreuse comme neige' : Exode 4,6];

- 'agah ['briller']: 'ôf tagahy'11 šalhebìytō' dnwreh ['Aussi brillera la flamme de son feu' : Job 18, 5] ;

Les verbes galīy ['se découvrir'], karīy ['être court], gardīy ['cesser'], mațīy ['arriver'], farah ['voler'] et damiy ['être semblable'] ont bien la première vocalisée d'un $a$, mais ils ne sont pas transitifs :

- galìy ['se découvrir'] : mețul dmen lwōty galìyty wasleqty ['Car d'auprès de moi tu t'es découverte et tu es montée' : Isaïe 57,8$]$;

- kariy ['être court']: wmețul dkariy mō'nō' wmeštīytō' qețnat ['Car la couche est courte et la couverture petite' : Isaïe 28,20$]$;

- gardìy ['être dépouillé, ôté' ${ }^{12}$ ] : wlō' temne' mōry bìšōtan dlö' mgarde' 'awlan menan ['Et ne tiens pas compte, Seigneur, de nos turpitudes, car notre iniquité ne peut être ôtée de nous'];

- mațīy ['arriver'] : zabnōtō sagìy'ōtō' lmawtōo mațīyt ['Bien des fois je suis parvenu jusqu'à la mort' : Ecclésiastique 34,13$]$;

- farah ['voler']: 'ayk șefrō' damfarhōo' 'al 'egōre' ['Comme le passereau qui vole sur les toits' : Psaume 102, 8] ; 
- damìy ['imiter, être semblable']: wa'ykō' damiyw 'ar'ōnōye' lašmayōne' ['Comment les êtres terrestres peuvent-ils être semblables aux célestes?'].

\section{Élucidation}

Il y a des cas où l'introduction des préfixes 'mnt et la vocalisation par a de la première quiescente amènent le verbe à un sens complètement étranger, comme :

- fra' hawbtō' ['il a rétribué un péché'] et 'afra', c'est-à-dire 'il a fait germer'.

- qbal ['accuser'], de qbwly $\bar{o}$, c'est-à-dire 'accusation' et 'aqbel, c'est-à-dire: 'rencontrer'.

- h̆far $g w b \bar{o}$ ['il a creusé une tombe'] et 'ahfar c'est-à-dire « avoir honte ».

Parmi les nombreux exemples donnés par BH, j'en conserverai deux qui portent sur la vocalisation en $a$ de la première consonne (équivalant à la FII $f a$ "ala de l'arabe) : šro $\bar{o}$ ('délier') et šarìy ('commencer') ; glō' ('découvrir, manifester') et galīy ('exiler').

On pourrait trouver un cas analogue en arabe moderne où 'adraba ('faire grève') est tout à fait étranger au sens de daraba ('frapper'), mais ici l'explication par le calque sur l'anglais résout le problème. Toutefois, on en trouve bien d'autres du même genre, qu'on ne saurait attribuer à un calque sur une langue étrangère, comme : șalā ('toucher, atteindre') et șallā ('prier, faire sa prière'); farața ('devancer') et farrața ('agir avec négligence, éloigner, détourner') ; qabila ('accueillir, recevoir') et qabbala ('donner un baiser') ; kalama ('blesser') et kallama ('adresser la parole à quelqu'un').

\section{Les verbes labiles}

BH aborde enfin la question des verbes labiles, ceux qui peuvent être transitifs et intransitifs, en gardant la même forme. Comme «ressuscite » : Jésus ressuscite/Jésus ressuscite Lazare.

Quatrième section : À propos des verbes qui sont à la fois transitifs et intransitifs

Ces verbes sont comme gaziy la'hrìn ['il a privé d'enfants un autre'] et gazìy $h w$ beh ['il s'est privé d'enfants lui-même']; ḩsan la'hrìyn ['il l'a emporté sur un autre'] et ḩsan hw beh ['il été fort en lui-même'].

À nouveau, BH multiplie les exemples et consacre même une demi-page à des attestations scripturaires dont nous allons rapporter quelques-unes particulièrement significatives :

- Pour le verbe hssan : transitif 'l'emporter sur' - wtar' 'e' dašywl lō' nehsnwnōh ['Les portes de l'enfer ne l'emporteront pas sur elle': Matth. 16,18]; intransitif "être dur, pénible » : wyeldat rōhệyl whesnat kad yōldō' ['Rachel accoucha et elle fut à la peine en accouchant' : Genèse 35,16$]$.

- Pour le verbe $n f a h$ : transitif 'souffler sur' - wkad nfah bhwn 'emar ['Et après avoir soufflé sur eux, il leur dit' : Jean 20, 22] ; intransitif 'gonfler, enfler' : wnefhat karsōh we'tmsiy 'ațōotōh ['Et son ventre enflera et ses os s'affaibliront' : Nombres, 5, 27].

- Pour le verbe 'amlek: transitif 'établir comme roi'-wa'mlek malkō' dBōbel laMtanyo' '['Et le roi de Babel établit comme roi Mattanya' : 2 Rois 24,17]; intransitif 'régner': mōryō 'amlek wga'ywtō' lbeš ['Le seigneur règne et il s'est vêtu de 
splendeur': Psaume 93, 1]. Il s'agit bien du même 'amlek qui dans un cas est transitif, 'nommer quelqu'un roi', et dans l'autre intransitif, 'régner'.

\section{Conclusion}

17 Si BH a emprunté le concept de transitivité à ZamaHšarī, il en a donné un traitement qui dépasse largement sa source. En effet, la seule préoccupation du grammairien arabe est d'assurer que tous les compléments sont bien à l'accusatif et d'identifier les causes de la transitivité. N'ayant pas ce problème d'assignement de l'accusatif, BH discute non seulement de la transitivité (simple, double ou triple) et de ses causes (en arabe, FIV et FII) comme le fait ZamaHš̌arī, mais en outre, il envisage aussi l'échec de ces causes : les cas où elles ne produisent pas la transitivité et les cas où elles produisent autre chose que de la transitivité, à savoir un sens nouveau étranger. Les mêmes échecs se produisent en arabe, mais ZamaȞšarī se garde bien d'en parler ici. Enfin, BH étudie en détail les verbes labiles qui sont à la fois transitifs et intransitifs, sujet que ZamaHš̌arī n'aborde pas.

En fait, ces phénomènes d'échec des causes de transitivité et de labilité ont bien été observés par les lexicographes arabes, entre autres par al-Sijistānī ( $255 \mathrm{H})$, al-Zajjāj (310 H) et al-Jawāliqī $(540 \mathrm{H})$. Le titre de ce dernier est particulièrement explicite : Mã jāa $a$ 'alā fa'altu wa-'af'altu bi-ma'nan wāhid ('Les cas où fa'altu et 'af'altu ont le même sens'). Il s'agit donc de faire l'inventaire des cas ou fa'ala (FI) et 'af'ala (FIV) ont un sens identique, comme : bašartu (FI) al-rajula, 'abšartuhu (FIV) et baššartuhu (FII) qui veulent tous dire : 'j'ai annoncé une bonne nouvelle à quelqu'un' (mot-à-mot 'quelque chose de réjouissant'). Dans sa préface, al-Zajjāj précise qu'il va parler :

- des verbes pour lesquels 'af'ala et fa'ala ont le même sens (voir l'exemple ci-dessus);

- des verbes où ils ont un sens totalement différent comme: dakartu (FI) al-šay'a 'j'ai mentionné la chose' et 'aḍkara (FIV) al-rajulu, 'l'homme a engendré un enfant mâle'; il s'agit ici d'un cas analogue à fra ' hawbtō 'il a rétribué un péché' et 'afra 'il a fait germer' de BH ;

- des cas où les deux formes étant attestées avec un sens identique, on préfère la forme IV comme dans 'abanna l-rajulu fi makān 'l'homme a résidé dans un lieu';

- des cas où l'on préfère la forme I, comme dans batartu al-šay'a : j'ai coupé la chose à la racine.

Mais les grammairiens n'ont pas intégré ces données à leur traitement. Jusque dans l'un des derniers avatars des théories des grammairiens arabes, le Ğāmi' al-durūs al-'arabiyya d'al-Ǵalāyīnī (1909), le traitement de la transitivité (I : 31-32) et celui de la forme IV ${ }^{13}$ (II : 224) ne mentionnent aucunement les données rebelles recueillies par les lexicographes. En revanche, $\mathrm{BH}$ propose un traitement qui intègre les règles et les exceptions, la grammaire et le lexique. Cela est cohérent avec son orientation vers les faits, qui se matérialise dans l'abondance des données, scripturaires ou non, citées par lui. 


\section{Sources primaires}

al-Ġalāyīnī, Musțafā. Ğāmi` al-durūs al- 'arabiyya. Sidon : Al-Maṭba'a al-'așriyya lil-ṭibā'a wal-našr. $13^{e}$ éd. 1978/1398.

al-Jawāliqī, Abū Manșūr. Mā jā'a 'alā fa'altu wa-'af'altu bi-ma'nan wāḥid, mu'allaf 'alā ḥurūf almu'jam. Éd. M. Ḥ. al-Dֵahabī. Damas : Dār al-fikr. 1982/1402.

al-Sijistānī, Abū Ḥātim, Fa'altu wa-'af 'altu. Éd. H.. I. al-'Ațiyya. Beyrouth : Dār Șādir. 1996/1416.

al-Zajjāj, Abū Isḥāq, Kitāb fa' altu wa-'af 'altu. Éd. M. Ḥ. al-Ḍahabī. Damas : al-Šarika al-muttaḥida lil-tawzī‘ . 1984/1404.

al-ZamaHš̌arī, Abū l-Qāsim. Al-Mufașṣal fì 'ilm al-'arabiyya. Beyrouth : Dār al-jīl. S.d.

Bar Hebræus, G. Ktōbō d-Ṣemhēe. Dans Le livre des splendeurs, la grande grammaire de Grégoire Bar Hebrceus. Skrifter utgivna av Kungl. Humanistiska vetenskapssamfundet i Lund, 4. Éd. par A. Moberg. Lund/Paris. C. W. K. Gleerup/Édouard Champion. 1922.

\section{Sources secondaires}

Bohas, G. 2003. Radical ou racine/schème. L'organisation de la conjugaison syriaque avant l'adoption de la racine. Le Muséon, $\mathrm{n}^{\circ}$ 116/3-4 : 343-376.

Bohas, G. 2008. Bar Hebraeus et la tradition grammaticale syriaque. Parole de l'Orient/Melto dMadndho, $\mathrm{n}^{\circ} 33: 145-158$.

Lallot, J. 1989. La grammaire de Denys de Thrace. Sciences du langage. Paris: Éd. du CNRS.

Merx, A., éd. 1889. Historia artis grammaticae apud Syros. Abhandlungen für die Kunde des Morgenlandes, 9/2. Leipzig : F. A. Brockhaus.

Moberg, A. 1907-1913, Buch der Strahlen: die grössere Grammatik des Barhebräus. Leipzig : O. Harrassowitz.

Thesaurus = Payne Smith, R., éd. 1879. Thesaurus Syriacus. 2 vol. Oxford : Clarendon Press.

\section{NOTES}

1. Voir Lallot (1989).

2. Voir la notice sur le Mufașsal dans le Corpus des textes linguistiques fondamentaux (http:// ctlf.ens-lyon.fr).

3. Chez ZamaHšarī (Mufașsal : 257), taHașșașa bi-l-fā'il ('concerne exclusivement le sujet').

4. Comme Bar Zo'bī, voir Bohas (2003).

5. On pourrait dire «transitiviser ", si le mot existait en français.

6. Équivalence déjà relevée par tradition syriaque antérieure (voir Bohas 2003).

7. BH cite les verbes à la troisième personne du singulier du passé qui est, pour lui, la forme de base du verbe. Je me conforme à l'usage occidental qui consiste à citer le verbe à l'infinitif. 
8. Le texte hébreu comporte le verbe tipqod. Dans ce contexte, il signifie 'reprocher'. Littéralement le sens du verset est donc en hébreu : «Et aujourd'hui tu me reproches une faute de femme ». En syriaque, la préposition 'al signifie 'sur' et aussi 'contre'. L'expression syriaque dkart 'lay ('tu te souviens contre moi') rend le verbe "tu me reproches». Je remercie David Hamidovic qui m'a fourni les précisions sur l'hébreu.

9. D'après l'exemple cité, c'est ce sens qui est concerné ici.

10. Contextualisé dans l'évangile, il s'agit précisément de 'vignerons'.

11. Il me semble qu'il y a ici un problème d'édition et que le verbe concerné est tgahe'y (pa“el avec la première vocalisée d'un $a$ ) 's'éteindra' et que le sens est : « Aussi se calmera la flamme de son feu ». Dans le Thesaurus, on trouve pour ce verset la traduction : sedabitur ardor ignis ejus. Mais alors il y a un problème de logique : que fait-il avec les verbes commençant par 'mnt?

12. Selon Mgr Feghali, e-mail du 29/12/2017, le mot mgared vient de l'arabe ğarrada 'enlever la peau, l'écorce'.

13. «La forme 'af'ala est le plus souvent pour la transitivité, c'est-à-dire pour transformer l'intransitif en transitif, et si le verbe est déjà transitif il devient doublement transitif. »

\section{RÉSUMÉS}

Cette contribution aborde la façon dont Bar Hebræus a emprunté au grammairien arabe ZamaHšarī la notion de transitivité et comment il l'a reformulée dans le cadre de sa grammaire du syriaque. Je procède en traduisant et commentant son texte et en comparant avec celui de ZamaHšarī. Son chapitre s'organise en quatre sections : 1 . Première section : à propos d'exemples de verbes intransitifs et transitifs; 2. Deuxième section: des causes de la transitivité; 3. Troisième section : à propos de l'échec des causes de la transitivité ; 4. Quatrième section : à propos des verbes qui sont à la fois transitifs et intransitifs. C'est dans ces deux dernières sections que se manifeste le mieux la différence entre les deux grammairiens ; et il apparaît que si Bar Hebræus a emprunté le concept de transitivité à ZamaHš̌arī, il en a donné un traitement qui dépasse largement sa source. En effet, la seule préoccupation du grammairien arabe est d'assurer que tous les compléments sont bien à l'accusatif et d'identifier les causes de la transitivité. N'ayant pas ce problème d'assignement de l'accusatif, Bar Hebræus discute non seulement de la transitivité (simple, double ou triple) et de ses causes (pour nous, FIV et FII) comme le fait ZamaHšarī, mais en outre, il envisage aussi l'échec de ces causes : les cas où elles ne produisent pas la transitivité et les cas où elles produisent autre chose que de la transitivité, à savoir un sens nouveau étranger. Les mêmes échecs se produisent en arabe. Enfin Bar Hebræus étudie en détail les verbes labiles qui sont à la fois transitifs et intransitifs.

This contribution deals with how Bar Hebræus borrowed the notion of transitivity from the grammarian of Arabic, ZamaHšsari, and how he reformulated it within his grammar of Syriac. I proceed by translating and commenting his text and comparing it with the text by ZamaHšrari. His chapter is organised into four sections: 1. First section: concerning examples of intransitive and transitive verbs; 2 . Second section: on the causes of transitivity; 3 . Third section: concerning the failure of the causes of transitivity; 4. Fourth section: concerning verbs which are both transitive and intransitive. The difference between the two grammarians is manifest in the final two sections in which it appears that although Bar Hebræus borrowed the concept of transitivity from ZamaHšarī, his treatment goes far beyond what is found in his source. Indeed, the only 
concern of the grammarian of Arabic is to ensure that all the complements are in the accusative and to identify the causes of transitivity. Without the problem of assigning the accusative, Bar Hebræus not only discusses transitivity (single, double or triple) and its causes (for us, FIV and FII) as ZamaHšarī does, but he also envisages the failure of these causes: the cases in which they do not produce transitivity and the cases in which they produce something different from transitivity, namely, a new foreign sense. Finally, Bar Hebræus studies in depth the labile verbs which are both transitive and intransitive.

INDEX

Mots-clés : Bar Hebræus, grammaire arabe, grammaire syriaque, transitivité, ZamaHšarī

Keywords : Arabic grammar, Bar Hebræus, Syriac grammar, transitivity, ZamaHšarī

\section{AUTEUR}

\section{GEORGES BOHAS}

ENS de Lyon, Interactions, corpus, apprentissage, représentations (UMR 5191 Icar), Lyon, France 